\title{
Aortic pressure wave reconstruction during exercise is improved by adaptive filtering: a pilot study
}

\author{
Wim J. Stok • Berend E. Westerhof • \\ Ilja Guelen · John M. Karemaker
}

Received: 14 February 2011/ Accepted: 18 June 2011/Published online: 1 July 2011

(c) The Author(s) 2011. This article is published with open access at Springerlink.com

\begin{abstract}
Reconstruction of central aortic pressure from a peripheral measurement by a generalized transfer function (genTF) works well at rest and mild exercise at lower heart rates, but becomes less accurate during heavy exercise. Particularly, systolic and pulse pressure estimations deteriorate, thereby underestimating central pressure. We tested individualization of the TF (indTF) by adapting its resonance frequency at the various levels of exercise. In seven males (age 44-57) with coronary artery disease, central and peripheral pressures were measured simultaneously. The optimal resonance frequency was predicted from regression formulas using variables derived from the individual's peripheral pressure pulse, including a pulse contour estimation of cardiac output (pcCO). In addition, reconstructed pressures were calibrated to central mean and diastolic pressure at each exercise level. Using a genTF and without calibration, the error in estimated aortic pulse pressure was $-7.5 \pm 6.4 \mathrm{mmHg}$, which was reduced to $0.2 \pm 5.7 \mathrm{mmHg}$ with the indTFs using pcCO for prediction. Calibration
\end{abstract}

Berend E. Westerholf and Ilja Guelen own shares of the BMEYE Company. BMEYE develops, manufactures and markets devices using Finapres Methodology ${ }^{\mathrm{TM}}$.

W. J. Stok $(\bowtie)$

Heart Failure Research Centre, Academic Medical Center,

University of Amsterdam, Room M01-215, Meibergdreef 9,

NL-1105 AZ Amsterdam, The Netherlands

e-mail: w.stok@amc.uva.nl

B. E. Westerhof · J. M. Karemaker

Heart Failure Research Centre, Academic Medical Center, University of Amsterdam, Room M01-216, Meibergdreef 9, NL-1105 AZ Amsterdam, The Netherlands

B. E. Westerhof · I. Guelen

BMEYE, Amsterdam, The Netherlands resulted in less scatter at the cost of a small bias $(2.7 \mathrm{mmHg})$. In exercise, the indTFs predict systolic and pulse pressure better than the genTF. This pilot study shows that it is possible to individualize the peripheral to aortic pressure transfer function, thereby improving accuracy in central blood pressure assessment during exercise.

Keywords Aortic pressure - Finger arterial pressure · Transfer function - Individualization

\section{Introduction}

The relevance of blood pressure changes during exercise is well established and is an independent predictor of cardiovascular morbidity and mortality [6, 13, 15]. Finger arterial blood pressure can easily be measured during exercise with the Finapres Methodology ${ }^{\mathrm{TM}}$, using the volume clamp method by Peńáz [18, 27] and the 'Physiocal' criteria by Wesseling [28]. However, changes in finger pressure during exercise are not representative of changes in aortic pressure. In general, systolic pressure is higher in peripheral arteries like the finger [3,5]. This physiological effect can also be observed in radial [19] and brachial arteries $[16,20]$. This amplification of systolic and pulse pressure changes with cardiovascular state, thus with workload and is different between subjects [24].

The purpose of this study is to improve aortic pressure reconstruction during exercise by use of adaptive filtering. Earlier, we found that the major change in the transfer function (TF) during exercise was the shift in peak resonance frequency (Fpeak) of the individual aorta-finger TF [24]. To individualize the TF (indTF), we looked for relations to predict this frequency shift from parameters extracted from the measured peripheral pressure pulse and 
patient characteristics. We used the dataset of seven cardiac patients as in our previous study $[3,24]$ to test if the bias and scatter in the reconstructed aorta pulse pressure are reduced with the indTF when compared to a general TF (genTF) [24].

\section{Methods}

\subsection{Patients}

The dataset was kindly provided by Dr. Blum. For detailed patient characteristics we refer to our earlier study [3, 24]. In short: seven cardiac patients, all males (Table 1), participated in an exercise protocol after selective coronary angiography. Nitrates, calcium channel blockers and $\beta$-blockers were taken by most of the subjects. Subject 1 was not under $\beta$-blockade and four subjects were moderately hypertensive.

The Ethics Committees of the Academic Hospital of the University of Utrecht (UMCU) and the Eemland Hospital in Amersfoort, the Netherlands, where the measurements were performed, approved the study. Informed consent was obtained from the subjects before the recordings were made.

\subsection{Measurements}

Ascending aortic pressure (AorP) was recorded with a calibrated tip manometer catheter (Millar type VPC 663). The measurement probe in this catheter is facing sideways, thus reducing kinetic effects during ejection. The catheter was inserted through the right brachial artery and located approximately $8 \mathrm{~cm}$ from the aortic valve. Calibrated finger arterial pressure (FinAP) was simultaneously measured with a Finapres model 5 (BMI, TNO, The Netherlands, formerly commercially available as the Ohmeda Finapres 2300 E NIBP). An appropriate size finger cuff was placed on the mid-phalanx of the middle finger of the left hand. Since hands were kept on the examination table, FinAP was corrected by subtracting $10 \mathrm{mmHg}$ from all recordings to compensate for the $10-15 \mathrm{~cm}$ difference between finger and heart level. This was shown to be appropriate on average [24].

\subsection{Exercise protocol}

Exercise was performed using an electromagnetically braked cycle ergometer with the subject in the supine position. The protocol consisted of incrementing the workload by $25 \mathrm{~W}$ every $2 \mathrm{~min}$, cycling at $70 \mathrm{rpm}$. The first step was 25 or $50 \mathrm{~W}$, depending on the expected maximal capacity of the subject, which had been tested before. Only one subject reached the maximal workload of $175 \mathrm{~W}$.

\subsection{Data analysis}

The FinAP and AorP signals were sampled at $100 \mathrm{~Hz}$, with $0.25 \mathrm{mmHg}$ resolution. Systolic and diastolic pressures and heart interval were calculated for each beat, using custommade software. Heart rate (HR) was determined from the interbeat interval and mean arterial pressures (MAPs) were calculated by integration over one beat and division by interbeat interval.

\subsection{Transfer function}

Data at rest and during the steady states of the exercise levels were selected for analysis. In most cases, more than 1 min of artifact-free continuous data was available. The minimum accepted steady state period was $10 \mathrm{~s}$; the maximum period was $60 \mathrm{~s}$. For each participant and for each exercise level the TF from aorta to finger was determined using an autoregressive exogenous (ARX) model method [4, 24]. This is a time domain method in which poles and zeros of a TF are adapted in a least square approach to approximate the desired output from the input signal. We used an implementation with ten poles and zeros. An average ARX TF was determined from the resting data of the seven subjects resulting in a multi-

Table 1 Subject data, maximal workload, HR and blood pressures during rest and at maximum workload

\begin{tabular}{|c|c|c|c|c|c|c|c|c|}
\hline \multirow[t]{2}{*}{ Subject } & \multirow[t]{2}{*}{ Age (years) } & \multirow[t]{2}{*}{ Weight (kg) } & \multirow[t]{2}{*}{ Height $(\mathrm{cm})$} & \multirow[t]{2}{*}{ Max load (W) } & \multicolumn{2}{|l|}{ Rest } & \multicolumn{2}{|c|}{ Maximal load } \\
\hline & & & & & HR (BPM) & MAP aorta $(\mathrm{mmHg})$ & HR (BPM) & MAP aorta $(\mathrm{mmHg})$ \\
\hline 1 & 44 & 81 & 177 & 125 & 80 & 93 & 152 & 132 \\
\hline 2 & 54 & 86 & 180 & 150 & 53 & 107 & 98 & 127 \\
\hline 3 & 49 & 76 & 176 & 75 & 86 & 99 & 120 & 98 \\
\hline 4 & 57 & 76 & 173 & 175 & 59 & 95 & 143 & 139 \\
\hline 5 & 42 & 83 & 172 & 150 & 87 & 120 & 146 & 149 \\
\hline 6 & 57 & 122 & 182 & 75 & 70 & 108 & 96 & 123 \\
\hline 7 & 50 & 85 & 176 & 150 & 73 & 116 & 113 & 131 \\
\hline
\end{tabular}

Values for main blood pressures (MAP) and HR are averaged over the data selected for calculating the TFs 
parameter average TF. The multi-parameter filter that is produced by the ARX method is less suitable for individualization. Therefore, a TF described by two 2 nd order filter sections (one high-pass and one low-pass), as was used by Gizdulich [9], was fitted to the frequency amplitude response of the average $\mathrm{TF}$, resulting in a characteristic genTF frequency response (Fig. 1). Using this approach, the lower, most important harmonics of the pressure wave are equally well processed as with the average TF. This genTF was then used as a starting point for the individualization. The TF was adapted so that the frequency of the peak (Fpeak) could be shifted to any desired frequency by lookup table, without affecting its gain.

Regression analysis was performed to develop a formula that could potentially predict the Fpeak to be used in each indTF. Data used for the prediction were subject parameters (age, height and weight), FinAP values (MAP, DBP, $\mathrm{HR}$ and $\max \mathrm{d} P / \mathrm{d} t$, the maximal value of the time derivative) and values derived from a pulse contour algorithm giving stroke volume (SV), cardiac output (CO) and total peripheral resistance (TPR) [10, 23]. In addition, foot-tofoot pressure wave delay between AorP and FinAP was incorporated, as this parameter may also be estimated (see 'Discussion') non-invasively and is a direct indicator of a change in wave transmission [30]. As a suboptimal TF has the largest effect on the reconstructed systolic pressure, we avoided to include parameters in the regression models that have a direct relation to the finger systolic pressure.

The Fpeak predicted by the regression formulas was used to shift the frequency for the peak of the adaptable TF to give the indTF.

\subsection{Calculations}

Reconstructed AorP was calculated from FinAP using the genTF and the indTF. After application of the TF, the

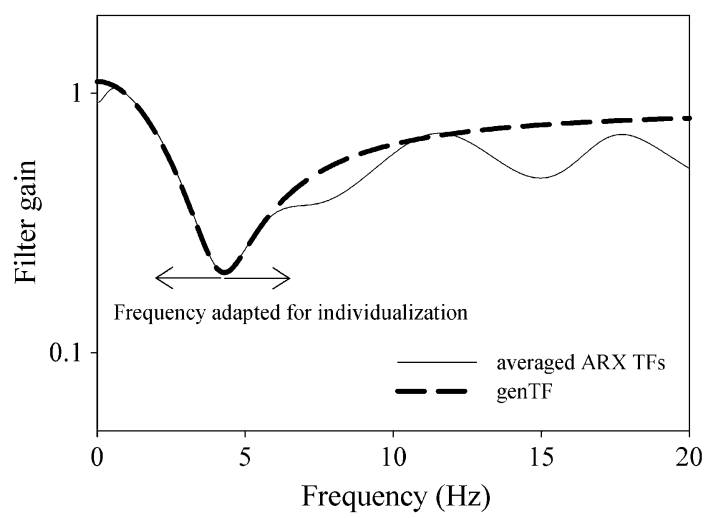

Fig. 1 Fit of genTF on the average of seven ARX reverse TFs at rest. ARX filters from Stok et al. [24] pressure wave was calibrated to central values of diastolic and MAP as is customary when a genTF is evaluated [12]. Although Finapres gives absolute pressure readings at the finger level, frictional losses and the pressure drop over the aorta to finger arterial segment may vary between subjects. When measuring an actual finger-aorta TF in a subject, these losses are incorporated in the TF as compensatory amplification. However, in case of a general reverse TF, the average value from the individual subjects at rest is incorporated in the filter. Therefore, theoretically, calibration is necessary for optimal results. In addition, this factor may not be stable during exercise. Both calibrated and noncalibrated values are therefore presented.

Reconstructed aortic pulse pressure (AorPP) using ind$\mathrm{TF}$ and genTF gave indAorPP and genAorPP, respectively. The averaged differences between the reconstructed AorPPs and measured AorPP were then used for the comparison of results from indTF and genTF. Results of all comparisons are presented in Bland-Altman plots [2].

Calculations were done with Matlab ${ }^{\mathrm{TM}}$ (The Mathworks, Inc) version R2006b. Multiple linear regression analysis was performed using SPSS 11.5.2. Ranges of remaining errors are given as well as means and standard deviations (mean $\pm \mathrm{SD}$ ).

\section{Results}

3.1 Prediction of the resonance frequency by regression analysis

Regression analysis showed that pulse contour-derived $\mathrm{CO}$ $[10,23](\mathrm{pcCO})$ as single parameter had the highest correlation with Fpeak (Fig. 2). The regression formula used to predict the Fpeak in the TF from pcCO was:

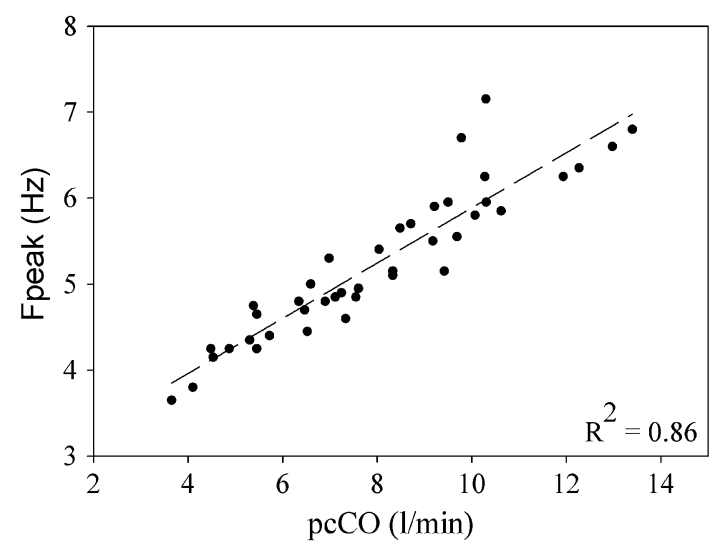

Fig. 2 Relation between resonance frequencies (Fpeak) during rest and exercise and pulse contour cardiac output (pcCO) calculated from the finger pressure wave 
Fpeak $=0.32 * \mathrm{pcCO}+2.68$.

Correlations between Fpeak and HR, dP/dt, SV, TPR, delay, MAP or DBP alone were weaker (Table 2), therefore these correlations were not further used for the prediction of Fpeak.

Multivariate stepwise regression models constructed with the TF peak frequency as the dependent variable resulted in regression Formulas 2 and 3, with and without pressure wave delay as parameter:

$$
\begin{aligned}
\text { Fpeak }= & 0.07 *(\mathrm{MAP}-\mathrm{DBP})+0.019 * \mathrm{HR}-0.13 \\
& * \text { Delay }+2.71 \\
\text { Fpeak }= & 0.07 *(\mathrm{MAP}-\mathrm{DBP})+0.023 * \mathrm{HR}+0.65 .
\end{aligned}
$$

As opposed to the Fpeak, we found no correlation between the peak amplitude (gain) of the original ARX TFs [24] measured during different workloads and the above parameters or with the available subject's biometric data. In addition, TF resonance peak amplitude did not change with increasing workload within any of the subjects. Therefore, peak amplitude was not considered in the individualization process.

\subsection{Aortic pressure wave reconstruction}

Differences between measured and reconstructed AorPP were reduced with the indTFs using any of the three regression formulas for prediction of the resonance peak. Formula 1 performed best with respect to precision although Formulas 2 and 3 were only marginally inferior. The incorporation of the AorP-to-FinAP pulse wave time delay in the regression, in addition to BP and HR, did not improve reconstruction. Table 2 gives the differences for rest and exercise combined using the genTF and the indTFs both without and with additional calibration. With a calibration for each level of exercise, precision improved using the indTFs, but a small bias was introduced. As can be observed in the corresponding Bland-Altman plots (Fig. 3a-d), the negative bias at the higher pulse pressures was reduced (for aorta pulse pressures above $70 \mathrm{mmHg}$, from -10.4 to $-0.8 \mathrm{mmHg}$ for the uncalibrated data).

Short traces of aortic pressure and reconstructed aortic pressure waves from three subjects are presented in Fig. 4, showing the wave reconstruction results using the genTF and the indTFs (Formula 1, pcCO) during rest and during each subject's maximal workload. Maximum HR was relatively high in subject 1 and 5, and low in subject 6 .

With the indTF, bias and precision improved and were well within Association for the Advancement of Medical Instrumentation (AAMI) criteria for bias and precision (bias, defined as mean difference, less than $5 \mathrm{mmHg}$ and precision, defined as SD of the difference, less than $8 \mathrm{mmHg}$ ) [1]. Results for the uncalibrated data with the genTF were outside the AAMI limits.

\section{Discussion}

In a small group of cardiac patients, individualized fingerto-aortic pressure TFs gave better pulse wave reconstruction during exercise than a genTF. Extra information from the peripheral pulse can aid in enhancing precision of

\begin{tabular}{|c|c|c|c|}
\hline \multirow[t]{2}{*}{ Variables } & \multirow[t]{2}{*}{$R^{2}$ Fpeak } & \multicolumn{2}{|c|}{ indAorPP error $(\mathrm{mmHg})$} \\
\hline & & No calibration & Workload calibration \\
\hline DBP & 0.35 & & \\
\hline MAP & 0.51 & & \\
\hline Delay & 0.56 & & \\
\hline TPR & 0.63 & & \\
\hline SV & 0.63 & & \\
\hline $\operatorname{Max} \mathrm{d} P / \mathrm{d} t$ & 0.69 & & \\
\hline HR & 0.71 & & \\
\hline $\mathrm{pcCO}$ & 0.86 & $0.2 \pm 5.7$ & $2.7 \pm 2.5$ \\
\hline $\mathrm{MAP}+\mathrm{DBP}+\mathrm{HR}+$ delay $($ Formula 2$)$ & 0.89 & $-0.4 \pm 5.8$ & $2.2 \pm 2.7$ \\
\hline MAP + DBP + HR (Formula 3) & 0.87 & $0.3 \pm 5.9$ & $2.8 \pm 2.9$ \\
\hline genTF & n.a. & $-7.5 \pm 6.4$ & $-3.9 \pm 5.4$ \\
\hline
\end{tabular}
central pressure wave reconstruction for different levels of

Table 2 Regression results and reconstructed pulse pressure errors

Regressions between TF resonance frequency (Fpeak) and measured and/or calculated parameters and three regression formulas, respectively. Errors between reconstructed (RecAorPP) and measured AorPP using the three regressions are given for no additional calibration and calibration at each workload. Results for the genTF are also given (Max $\mathrm{d} P / \mathrm{d} t$ is the maximal value of the time derivate of FinAP; MAP and DBP are FinAP mean and diastolic pressure; pcCO is pulse contour cardiac output; SV is stroke volume; TPR is total peripheral resistance; HR is heart rate and Delay is travel time between central and peripheral pulse wave) 

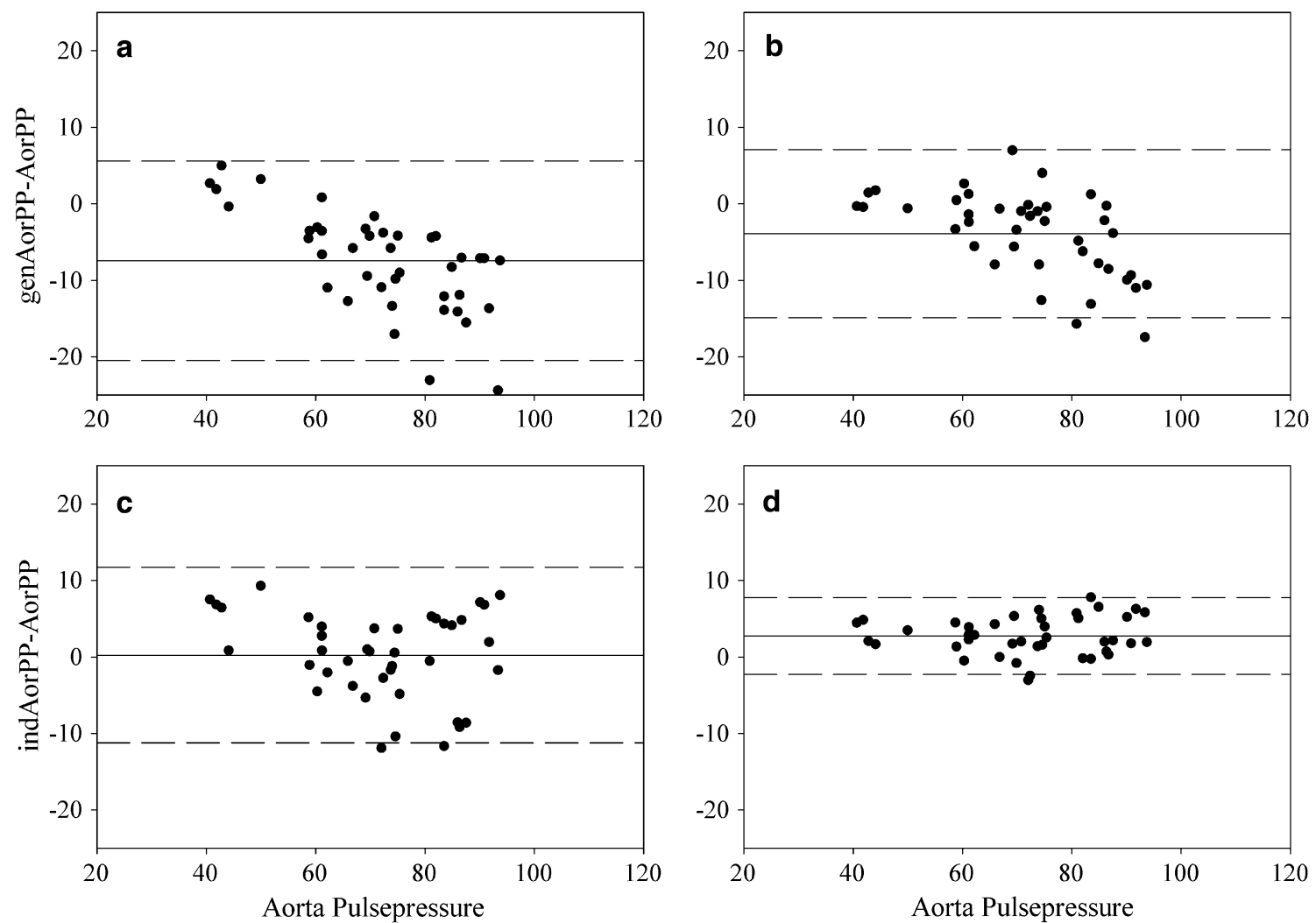

Fig. 3 Bland-Altman plots (average \pm 2 SD) plot of measured aorta pressure and reconstructed pulse pressure with the genTF (genAorPP, top) and the individual TFs (indAorPP, bottom). Results without (a,

c) and with (b, d) additional calibration. Points are results from all measured workloads in seven subjects

Fig. 4 Reconstruction (thin lines) of aorta pressure waves (bold lines) from finger pressure during rest and at maximum workload in three subjects. From left to right: genTF in rest and at maximal workload and with individual TFs (indTF) using finger $\mathrm{pcCO}$ in rest and at maximal workload. Subject 1 and 5 with relatively high maximum HR, subject 6 with low maximum HR
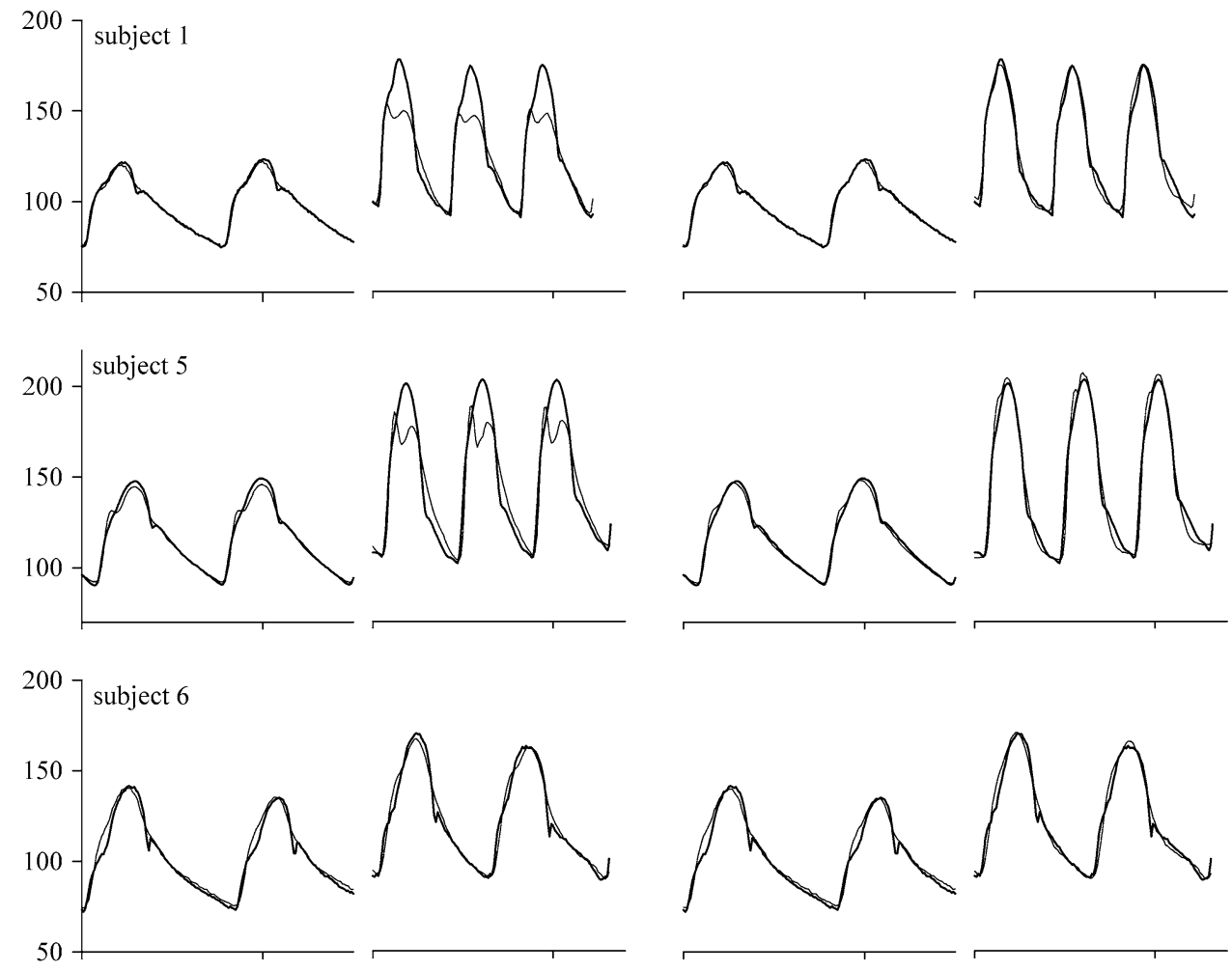

Time (s) 
exercise. This information can be easily and noninvasively obtained during a measurement, i.e., pulse contour (CO) or peripheral mean and diastolic arterial pressure and HR. In this study, data from only seven subjects were available as 'trainings set'; therefore we could not evaluate our concept on a separate 'test set' group. However, these first results are promising.

During exercise, central pressure reconstruction from peripheral measurements is challenging due to the combined effects of shifting of the actual TF's resonance peak (Fpeak) to higher frequencies and the shift in harmonics of the pressure wave with increased HR. In an earlier study, we found that a genTF underestimated central pressures at higher intensities of exercise in a number of the subjects, although it performed well at rest [24]. Similarly, Westerhof et al. [31] concluded that individualization did not improve the aortic wave reconstruction in patients at rest. In the exercise study by Sharman [22], where peripheral pressure (radial) was measured by tonometry at one exercise level (light to moderate), it was concluded that a genTF may be used during exercise. In our earlier study [24], we found a considerable spread in the reconstructed pressures during exercise which can be attributed to a higher maximum workload in our study (increases in MAP $+25 \pm 16$ vs. $+20 \pm 10 \mathrm{mmHg}$ and in $\mathrm{HR}+51 \pm 21$ vs. $+15 \pm 7$ BPM) when compared with the study of Sharman. In effect, in the study of Sharman there was also a tendency to underestimate systolic pressure $(-4.7 \pm 3.3 \mathrm{mmHg})$ with the largest deviations at the higher pressures. Here, we show that reconstructed pressure can be improved with adaptive filtering.

The use of genTFs is well accepted [7]. Several methods to individualize aortic-peripheral TFs have been proposed, mostly by model fitting, but without definite success. Segers [21] did not find a relation between the fitted parameters of a transmission line model of the upper limb and the physiological subject parameters. Sugimachi [25] used transfer delay for the individualization, but without significant improvement over a genTF, contrary to what would be expected from oscillatory flow theory [16, 29, 30]. A recently proposed method uses time delay as well as the knowledge that aortic flow is zero during diastole [26]. Karamanoglu [11] used adaptive filtering of the finger pressure wave making use of simultaneously derived carotid tonometric data. However, the latter is difficult to perform during exercise because of movement artifacts and it is unknown whether the aortic to carotid TF will be stable under those circumstances.

In this study, we proposed individualization by direct statistical correlation between one of the main features of the TF and physiological subject parameters, both by using the inter-subject variability and by introducing exercise evoked changes within each subject. Parameters were chosen that could easily be extracted form the finger pressure signal. The applied pcCO algorithm relates the pressure systolic area (PSA) to flow by an estimate of impedance, incorporating corrections for age, MAP and HR $[10,23]$. PSA alone did not correlate with Fpeak $\left(R^{2}=0.027\right)$. In addition, pressure wave delay between aorta and finger was incorporated on theoretical grounds $[16,25,30]$. This delay can be measured noninvasively using the ECG, but appropriate corrections for changes in pre-ejection period have to be made. However, time delay did not improve reconstruction over and above the more easily obtained pcCO.

During exercise, two major changes occur in the hemodynamic status of a subject: both HR and mean blood pressure increase. HR has no direct effect on pressure transfer, although some authors $[8,14,32]$ mention an effect on distensibility or on pulse wave velocity independent of a change in pressure. Increased mean blood pressure will increase wave speed, which has a direct effect on travel time over the arteries and on the TF [30]. Travel time is reduced and the resonance peak of the pressure TF will shift to higher frequencies [16] as was confirmed in our earlier study [24].

Due to the increased HR during moderate to high workloads the harmonics of the pressure wave also shift to higher frequencies. The combined effect of changes in TF and HR makes the result of AorPP reconstruction with a genTF during exercise rather unpredictable.

The relevance of an increase in HR and the shift in Fpeak in relation to an underestimation of reconstructed pulse pressure can be understood when considering the harmonics of the pressure wave involved. At resting HR, the TF's Fpeak (in our subjects on average at $4.25 \mathrm{~Hz}$ ) was between the 3rd and 4th harmonic of the pressure wave, whereas most of the energy is in the 1st and 2nd harmonic. With increasing workload and HR, both the TF's Fpeak and the HR harmonics are shifted to higher frequencies [24]. More so for the HR harmonics, but still mainly the higher harmonics are amplified on transmission towards the periphery. In this situation, a general reverse TF, optimized for the resting situation, will reduce the amplitude of the lower two harmonics, leading to a distortion of the reconstructed wave. A change in TF without a change in HR (as under complete autonomic blockade) will have a much lower impact, as only higher harmonics are involved. Pulse pressure will be underestimated to variable extents, an effect also described for the period directly post-exercise in a recent study by Payne [17]. These effects may reduce the applicability of a genTF, although at low exercise loads acceptable results may be obtained [22, 24].

With the individualized TF as proposed in this study, bias and scatter are reduced, such that central blood pressure can be reliably determined in an individual patient 
even at higher workloads. The results were well within the AAMI criteria [1].

\subsection{Limitations}

We were not able to test the concept in a separate control group. Therefore, this must be considered a pilot study in a small but important patient group. A possible age dependency was not investigated since we considered our group too homogeneous in that respect nor were we able to assess a gender-related difference.

In practical use during exercise, additional calibration will be difficult to achieve. However, even without calibration, the bias at higher pulse pressures is much reduced.

All but one subject were under $\beta$-blockade, therefore HRs were relatively low (Table 1), but increased in all subjects in parallel and maximum increase in HR was directly related to the maximum workload. We found no relation between the shift in Fpeak and the use of a $\beta$-blocker [24].

\subsection{Perspectives}

We consider that our results may form a basis for further research to improve the individualization of the fingeraorta TF. Only peripherally and non-invasively recorded data were used to individualize the TF, ensuring wide applicability. The proposed method can easily individualize a finger-to-aortic pressure TF, improving accuracy and increasing the reliability in individual patient's blood pressure measurements during exercise.

Acknowledgments We are indebted to Dr. Viktor Blum for providing the original data set [3].

Disclosure Patent has been applied for the principle described in this manuscript.

Open Access This article is distributed under the terms of the Creative Commons Attribution Noncommercial License which permits any noncommercial use, distribution, and reproduction in any medium, provided the original author(s) and source are credited.

\section{References}

1. AAMI (2002) American national standard for electronic or automated sphygmomanometers. Association for the Advancement of Medical Instrumentation, Arlington, VA

2. Bland JM, Altman DG (1986) Statistical methods for assessing agreement between two methods of clinical measurement. Lancet $1: 307-310$

3. Blum V, Carriere EG, Kolsters W, Mosterd WL, Schiereck P, Wesseling KH (1997) Aortic and peripheral blood pressure during isometric and dynamic exercise. Int J Sports Med 18:30-34
4. Chen CH, Nevo E, Fetics B, Pak PH, Yin FC, Maughan WL, Kass DA (1997) Estimation of central aortic pressure waveform by mathematical transformation of radial tonometry pressure. Validation of generalized transfer function. Circulation 95: $1827-1836$

5. Eckert S, Horstkotte D (2002) Comparison of Portapres noninvasive blood pressure measurement in the finger with intraaortic pressure measurement during incremental bicycle exercise. Blood Press Monit 7:179-183

6. Filipovsky J, Ducimetiere P, Safar ME (1992) Prognostic significance of exercise blood pressure and heart rate in middle-aged men. Hypertension 20:333-339

7. Gallagher D, Adji A, O'Rourke MF (2004) Validation of the transfer function technique for generating central from peripheral upper limb pressure waveform. Am J Hypertens 17:1059-1067

8. Giannattasio C, Vincenti A, Failla M, Capra A, Ciro A, De Ceglia S, Gentile G, Brambilla R, Mancia G (2003) Effects of heart rate changes on arterial distensibility in humans. Hypertension 42: 253-256

9. Gizdulich P, Prentza A, Wesseling KH (1997) Models of brachial to finger pulse wave distortion and pressure decrement. Cardiovasc Res 33:698-705

10. Jansen JRC, Wesseling KH, Settels JJ, Schreuder JJ (1990) Continuous cardiac output monitoring by pulse contour during cardiac surgery. Eur Heart J 11(Suppl I):26-32

11. Karamanoglu M (2004) A method for estimation of intensity of wave reflection in an elastic tube. Cardiovasc Eng 4:229-236

12. Kelly R, Fitchett D (1992) Noninvasive determination of aortic input impedance and external left ventricular power output: a validation and repeatability study of a new technique. J Am Coll Cardiol 20:952-963

13. Kurl S, Laukkanen JA, Rauramaa R, Lakka TA, Sivenius J, Salonen JT (2001) Systolic blood pressure response to exercise stress test and risk of stroke. Stroke 32:2036-2041

14. Lantelme P, Mestre C, Lievre M, Gressard A, Milon H (2002) Heart rate: an important confounder of pulse wave velocity assessment. Hypertension 39:1083-1087

15. Mundal R, Kjeldsen SE, Sandvik L, Erikssen G, Thaulow E, Erikssen J (1996) Exercise blood pressure predicts mortality from myocardial infarction. Hypertension 27:324-329

16. Nichols WW, O'Rourke MF (2005) McDonald's blood flow in arteries: theoretical, experimental and clinical principles, 5 th edn. Hodder Arnold, London

17. Payne RA, Teh CH, Webb DJ, Maxwell SR (2007) A generalized arterial transfer function derived at rest underestimates augmentation of central pressure after exercise. J Hypertens 25:2266-2272

18. Peñáz J (1973) Photoelectric measurement of blood pressure, volume and flow in the finger. In: Digest of the 10th International Conference on Medicine and Biological Engineering, p. 104

19. Rowell LB, Brengelmann GL, Blackmon JR, Bruce RA, Murray JA (1968) Disparities between aortic and peripheral pulse pressures induced by upright exercise and vasomotor changes in man. Circulation 37:954-964

20. Segers P, Stergiopulos N, Verdonck P, Verhoeven R (1997) Assessment of distributed arterial network models. Med Biol Eng Comput 35(6):729-736

21. Segers P, Carlier S, Pasquet A, Rabben SI, Hellevik LR, Remme E, De Backer T, De Sutter J, Thomas JD, Verdonck P (2000) Individualizing the aorto-radial pressure transfer function: feasibility of a model-based approach. Am J Physiol Heart Circ Physiol 279:H542-H549

22. Sharman JE, Lim R, Qasem AM, Coombes JS, Burgess MI, Franco J, Garrahy P, Wilkinson IB, Marwick TH (2006) Validation of a generalized transfer function to noninvasively derive central blood pressure during exercise. Hypertension 47:1203-1208 
23. Stok WJ, Baisch F, Hillebrecht A, Schulz H, Meyer M, Karemaker JM (1993) Noninvasive cardiac output measurement by arterial pulse analysis compared with inert gas rebreathing. J Appl Physiol 74:2687-2693

24. Stok WJ, Westerhof BE, Karemaker JM (2006) Changes in finger-aorta pressure transfer function during and after exercise. J Appl Physiol 101:1207-1214

25. Sugimachi M, Shishido T, Miyatake K, Sunagawa K (2001) A new model-based method of reconstructing central aortic pressure from peripheral arterial pressure. Jpn J Physiol 51:217-222

26. Swamy G, Xu D, Olivier NB, Mukkamala R (2009) An adaptive transfer function for deriving the aortic pressure waveform from a peripheral artery pressure waveform. Am J Physiol Heart Circ Physiol 297:H1956-H1963

27. Wesseling KH (1991) Finapres, continuous non-invasive finger arterial pressure based on the method of Peñáz. In: Meyer-Sabellek W, Anlauf M, Gotzen R, Steinfeld L (eds) Blood pressure measurements. Steinkopff Verlag, Darmstadt, pp 161-172
28. Wesseling KH, De Wit B, Van der Hoeven GMA, Van Goudoever J, Settels JJ (1995) Physiocal, calibrating finger vascular physiology for Finapres. Homeostasis 36:67-82

29. Westerhof BE (2005) Blood pressure analysis on time scales from seconds to days. University of Amsterdam, Amsterdam

30. Westerhof BE, Guelen I, Stok WJ, Wesseling KH, Spaan JA, Westerhof N, Bos WJ, Stergiopulos N (2007) Arterial pressure transfer characteristics: effects of travel time. Am J Physiol Heart Circ Physiol 292:H800-H807

31. Westerhof BE, Guelen I, Stok WJ, Lasance HA, Ascoop CA, Wesseling KH, Westerhof N, Bos WJ, Stergiopulos N, Spaan JA (2008) Individualization of transfer function in estimation of central aortic pressure from the peripheral pulse is not required in patients at rest. J Appl Physiol 105:1858-1863

32. Wilkinson IB, Mohammad NH, Tyrrell S, Hall IR, Webb DJ, Paul VE, Levy T, Cockcroft JR (2002) Heart rate dependency of pulse pressure amplification and arterial stiffness. Am J Hypertens 15:24-30 